\title{
Megaproyectos como productores de suelo urbanizable. Análisis de tres casos de estudio en Hermosillo, Sonora (2004- 2015).
}

Megaprojects as producers of developable land. Analysis of three case studies in Hermosillo, Sonora (2004-2015).

Recibido: $28 / 03 / 2017$

Aceptado: 10/02/2017

Brisa Violeta Carrasco Gallegos ${ }^{1}$

Yanely Consuelo Estrada Santoyo ${ }^{2}$

\section{Resumen:}

Los megaproyectos en México se han presentado como una de las principales posibilidades para ejercer el crecimiento y desarrollo de las ciudades. Hoy por hoy, las ciudades medias también se encuentran en la disyuntiva por apuntar hacia el desarrollo de grandes proyectos urbanos, que operan como los principales generadores de atractivo y multiplicación de capitales en torno al espacio urbano. El caso de Hermosillo, Sonora ubicado en el noroeste de México, señala un proceso de expansión y rehabilitación urbana a partir de la generación de proyectos urbanos a gran escala en los últimos quince años. Su impacto se ha reflejado en la acrecentando producción de suelo urbanizable vinculado al desarrollo inmobiliario de viviendas residenciales. El objetivo del siguiente artículo es evidenciar la morfología urbana de Hermosillo, a partir de hacer un análisis histórico descriptivo que nos permita identificar las zonas urbanas, así como sus consecuencias. La originalidad del trabajo se centra en la falta de estudios que aborden la problemática desde la dimensión urbana para el caso, así la capacidad de demostrar que los megaproyectos urbanos han carecido de una verdadera posibilidad para el desarrollo y, por ende, han generado la inserción de otras problemáticas en torno a la ciudad.

\section{Palabras Clave:}

Megaproyectos urbanos; Morfología urbana;

Desarrollo inmobiliario.

\begin{abstract}
The megaprojects in Mexico have been presented as one of the main possibilities to exert the growth and development of the metropolitan cities. Nowadays, medium-sized cities are also in the dilemma for pointing to the development of large urban projects, which operate as the main generators of attractiveness and multiplication of capitals around the urban space. The case of Hermosillo, Sonora, located in the northwest of Mexico, indicates a process of urban expansion and rehabilitation from the generation of large-scale urban projects in the last fifteen years. Its impact has been reflected in the increase in the production of urban land linked to the development of residential real estate. The objective of the following article is to show the urban morphology of Hermosillo, from a descriptive historical analysis that allows us to identify urban areas as well as their consequences. The originality of the work is centered in the lack of studies that address the problem from the urban dimension for the case, as well as the capacity to demonstrate that urban megaprojects have lacked a real possibility for development and, therefore, have generated the insertion of other problems around the city.
\end{abstract}

\section{Key words:}

Urban megaprojects; urban morphology; real state development

\footnotetext{
${ }^{1}$ Nacionalidad: mexicana. Doctora en Ciencias Sociales; Facultad de Geografía Universidad Autónoma del Estado de México. brisavioletac@hotmail.com ${ }^{2}$ Nacionalidad: mexicana. Doctorando en Ciencias Sociales. El Colegio de Sonora yanely.estrada.santoyo@gmail.com
} 


\section{Introducción}

Las transformaciones en la ciudad operan a razón los aspectos económicos, políticos, sociales y culturales de una sociedad. Los megaproyectos urbanos han resultado ser una de las formas de intervenir la ciudad en nuestros tiempos. Su aplicación tiene como objetivo generar mercados de consumo inmobiliario dentro de la ciudad.

La competencia por construir el megaproyecto urbano se intensifica todos los días: túneles que se construyen por debajo del mar, el rascacielos más alto, el edificio más grande, archipiélagos artificiales destinados a actividades recreativas como el turismo, ciudades ecológicas, inteligentes, tecnológicas, entre otros muchos más; son sólo una muestra de la capacidad del hombre por emprender el viaje hacia el ideal urbano como un destino mejor.

Los megaproyectos urbanos se encuentran buscando satisfacer mercados, competencia y generar grandes ganancias: en el caso de América Latina ha conformado una serie de proyectos que han consolidado áreas urbanas en las ciudades, generando nuevas apuestas hacia el desarrollo y el crecimiento urbano a partir de inducir a la generación de suelo urbanizable.

En el caso mexicano se han multiplicado principalmente en sus metrópolis, para ahora replicar en ciudades medias, en vías de crecimiento y expansión urbana. Así es como surge Hermosillo, Sonora en donde la diversidad de espacios ha generado la proliferación de proyectos urbanos a partir de cada administración gubernamental.

Sus consecuencias se reproducen a partir de espacios urbanos, en los que abundan la saturación de zonas comerciales y residenciales, que si bien van configurando el paisaje urbano de acuerdo a nuevos mercados y expectativas de los consumidores tanto locales como foráneos.
La necesidad de poner en evidencia el desarrollo y el crecimiento de las ciudades en torno a los megaproyectos urbanos se torna de relevancia en la actualidad, debido a la multiplicidad de problemas urbanos que carecen de atención inmediata (vialidades, seguridad, pavimentación y saneo en las calles). Este hecho sin duda pone en tela de juicio la pertinencia de llevar a cabo proyectos de esta índole, así como las condiciones bajo las cuáles se vienen haciendo las ciudades en México en la actualidad.

A continuación, se presenta un acercamiento a la definición de los megaproyectos urbanos, una breve reseña de los megaproyectos urbanos en México, y por último el caso de Hermosillo, desde un análisis histórico descriptivo los principales megaproyectos urbanos y sus principales consecuencias en la morfología urbana en los últimos quince años.

\section{Hacia una definición de los megaproyectos urbanos}

En aras a proponer una definición de los megaproyectos, parten de la capacidad de transformación de los entornos donde son desarrollados, así como para involucrar diversos sectores de la sociedad. En ese sentido, se retoma la definición de la Administración Federal de Carreteras de Estados Unidos, quienes consideran megaproyecto a cualquier obra de infraestructura cuyo costo supera mil millones de dólares, con un importante nivel de atracción pública, interés político y presupuestos públicos. No obstante, la construcción del concepto deriva en buena parte a las condiciones geográficas, políticas y económicas, es decir el contexto donde este se desarrolla (Talledos Sánchez e Ibarra García, 2016).

En un acercamiento certero en torno a los megaproyectos urbanos es necesario resaltar aquellas características que inducen a su denominación, que sin duda se encuentran vinculados a sus efectos y sus consecuencias en la trama 
urbana. Dentro de las aportaciones a una definición de los megaproyectos urbanos se encuentran dos corrientes, una originada en los trabajos de Mario Lungo (2005a, 2005b), Cuenya (2009) y Cuenya y otros (2012) con su perspectiva desde Latinoamérica denominándolos grandes proyectos urbanos. Por otro lado, los estudios de los megaproyectos urbanos en EUA y Europa surgen a partir de los trabajos de Faisntein (2009) y Díaz Orueta (2009, 2015). A continuación, analizaremos ambas propuestas.

Los principales estudios latinoamericanos surgen a partir de Lungo (2005a, 2005b), quien desde su propuesta nos acerca a la denominación de grandes proyectos urbanos. Estos se integran a partir de no solamente grandes obras emblemáticas (como el mismo autor, denomina a los megaproyectos urbanos realizados principalmente en Europa), también incluye grandes operaciones urbanas así como acciones urbanas en conjunto, que por su capacidad de integración tienen un importante impacto en la transformación de las ciudades. ${ }^{1}$

El término grandes proyectos urbanos, Cuenya (2009) propone nombrar de dicha manera; aquellas megas operaciones de renovación urbana desde la iniciativa pública, las cuáles engloban un importante componente de actividades del sector terciario a partir del financiamiento en infraestructuras y edificios. De ahí que su primera propuesta de tipologías de grandes proyectos urbanos Señalando intervenciones urbanas de redesarrollo, re funcionalización y expansión de zonas urbanas.
Para la propuesta de Cuenya y otros (2012), el abordaje de los grandes proyectos urbanos es generado desde una perspectiva más compleja desde su dimensión funcional, en donde las intervenciones urbanas encierran una serie de usos jerárquicos del espacio, generando nuevas centralidades derivadas de su localización estratégica. Aunadas a la demanda del poder adquisitivo más alto, sus tipologías se expanden a operaciones no sólo a zonas de renovación y rehabilitación urbana, sino también operan a partir del montaje de infraestructura (edificios) con el fin de albergar grandes eventos de índole cultural y deportivo.

Faisntein (2009) y Díaz Orueta $(2009,2015)$ manejan una serie de coincidencias en sus propuestas en torno a los megaproyectos urbanos. En cuanto a su definición, consideran dos tipos de intervenciones urbanas; a) proyectos urbanos vinculados a generar emblemas a partir de la arquitectura (edificios con fuerte carga simbólica) y b) esquemas urbanos que conjuntan usos residenciales, de servicios, industria, medios de transporte, turísticos, entre otros.

Entre las características que surgen en torno a la definición viene la singularidad de los aspectos económicos y políticos, como los principales agentes en la toma de decisiones en torno a los megaproyectos urbanos. Por ello, sus consecuencias sociales y ambientales en las ciudades en donde son desarrollados generan poca o nula relación con los deseos e intereses de los habitantes. ${ }^{2}$

\footnotetext{
${ }^{1}$ Desde su perspectiva, es difícil plantear una propuesta operable para los grandes proyectos urbanos que permita considerar su escala y tipología, a lo cual propone plantear su abordaje desde la complejidad que genera en términos sociales y económicos. Para ello, resulta necesario considerar el potencial de los grandes proyectos urbanos para estimular el desarrollo en las ciudades a partir de su vocación urbana, su carácter estratégico y su capacidad para ofertar las características del lugar.

${ }^{2}$ El estudio entorno a los megaproyectos urbanos se ha encontrado inmerso en diversas perspectivas desde las económicas hasta antropológicas, hecho que ha permitido tener un conocimiento más complejo acerca de su desarrollo e impactos en las ciudades principalmente norteamericanas y europeas. No obstante, las diferencias entre ambos radican en su forma de operar; en tanto, para el caso estadounidense la operación de megaproyectos urbanos se enfoca en la creación de mega estructuras urbanas (puentes, carreteras, aeropuertos, y otros) mientras para el caso europeo emergen con el objetivo de generar grandes emblemas arquitectónicos en torno a la ciudad.
} 
Por otro lado, el aspecto cultural resulta clave para considerar los megaproyectos urbanos como una atracción urbana que pueda radiar hacia el turismo, a partir de actividades deportivas por medio de estadios o si bien, exposiciones de arte mediante museos. El fin justifica los medios, por tal motivo es necesario demostrar su necesidad a partir de su promoción entre la población como parte de un proyecto que traerá grandes beneficios primordialmente a los residentes.

La discusión en torno a la definición en cuanto a grandes proyectos urbanos o bien, megaproyectos urbanos aplica en distinción en relación a la localización de los casos examinados. No obstante, ambas propuestas mantienen significativos aportes para la observación y la posible identificación, así como categorización de los proyectos urbanos de acuerdo a las características y tipologías propuestas. En el afán de generar una definición, caracterización y tipología que pueda aportar a nuestro caso de estudio habremos de considerar ambas posturas.

\section{Megaproyectos urbanos en México}

Los escenarios para la generación de megaproyectos urbanos en México, han sido el clima económico y los beneficios en pos del desarrollo que auguran a partir de su construcción e inversión. La intensificación de una serie de reformas neoliberales conforme la entrada en vigor del Tratado de Libre Comercio de América del Norte (TLCAN), conllevó a una mayor apertura al comercio internacional, la desregulación de la actividad económica, la privatización de las empresas del Estado, así como la descentralización de la gestión pública (Garza y otros, 2003).
Esta serie de hechos trajeron consigo la dinamización del sector inmobiliario a partir de capitales privados, nacionales y extranjeros principalmente en Ciudad de México y el resto de la Zona Metropolitana del Valle de México (Pradilla 2004, 57). ${ }^{1}$ Bajo estas condiciones políticas y económicas, comenzaron por generar grandes proyectos urbanos destinados a la renovación o recuperación de zonas históricas, la construcción de grandes torres de oficinas y hoteles, centro comerciales y tiendas departamentales, los cuáles fueron consolidando importantes corredores urbanos destinados al sector terciario (Pradilla 2004, 57).

Todos los proyectos urbanos mencionados presentaron una serie de condiciones sociales y efectos urbanos en común. Emergieron ante la ausencia de la participación de actores ciudadanos, si bien, aunque fueron planteados bajo el discurso de equidad, las condiciones resultaron adversas para los habitantes. En consecuencia, la Ciudad de México fue generando problemas urbanos tales como; segregación, polarización y fragmentación derivada de la intensificación del sector terciario en ciertas zonas de la ciudad (Pradilla 2004).

Uno de los grandes proyectos urbanos con mayor impacto en la Ciudad de México, es sin duda, Santa Fe. Ubicado en las afueras de la ciudad, surge a finales de los años ochenta como un megaproyecto urbano determinado a promover la inversión inmobiliaria a nivel global, a partir de generar un conjunto urbano que permitiera alojar a grandes compañías internacionales (Moreno Carranco, 2008). En sí, un centro corporativo inmobiliario con capacidad de recuperar las zonas más deterioradas de la ciudad, a partir de impulsar la actividad económica terciaria planteando un nuevo modelo de ciudad con el

\footnotetext{
${ }^{1}$ Es importante señalar que previamente en otras zonas del país, habían emergido una serie de megaproyectos urbanos enfocados a detonar el turismo denominados como Centros Integralmente Planeados. Surgen como experiencia Acapulco en los años cincuenta y Cancún proyectado en los años setenta, como los principales íconos del turismo mexicano. Hecho que trajo consigo una serie de desarrollos inmobiliarios enfocados principalmente al sector hotelero o bien de servicios. Las ciudades comenzaron a sentir los estragos a partir de problemáticas urbanas como privatización de las playas y espacios públicos, segregación socio-espacial y principalmente, la gestión del espacio urbano a partir de las dinámicas de la demanda del mercado inmobiliario extranjero.
} 
mayor desarrollo urbano en América Latina (Valenzuela, 2007).

El proyecto fue diseñado como un complejo urbano integrado por edificios de oficinas corporativas, conjuntos residenciales, centros comerciales, de convenciones y de entretenimiento. $\mathrm{El}$ proyecto fue realizado por arquitectos de origen mexicano; tales como Ricardo Legorreta, Teodoro González de León y Abrahan Zabludovsky. No obstante, a los años fueron incorporándose obras de arquitectos reconocidos a nivel internacional (Moreno Carranco, 2008).

Santa Fe se presentó como un caso paradigmático de los megaproyectos urbanos en México, tanto por su diseño y su gestión urbana (Valenzuela, 2013). Dando los primeros indicios de la neoliberación urbana a partir de su perfil para administrar recursos tanto públicos como privados, en torno a la construcción de la ciudad y la inversión inmobiliaria. Se convirtió en un modelo urbano a seguir particularmente para las zonas metropolitanas del país.

En Guadalajara, Jalisco conformada a partir de una zona metropolitana, ubicada en la zona occidente del país, se han organizado a lo largo de su historia urbana, una serie de estrategias enfocadas a participar en los procesos económicos del país. Hecho que la ha colocado como una de las ciudades más importantes de México, debido a su alta capacidad de atracción de capitales a partir de la industria y la comercialización de productos a nivel internacional (Rivera Borrayo y otros, 2009).

Por ende, los grandes proyectos urbanos se presentaron como un hecho en cadena a los generados en la Ciudad de México. Su registro se ha convertido en uno de los campos aún abierto para la investigación urbana, no obstante, entre los megaproyectos urbanos analizados se encuentran los siguientes; a) Centro de Cultura, Convenciones y Negocios JVC, el cual integra once edificios a partir de oficinas, centros comerciales y de entretenimiento, entre otros. Incorpora una serie de diseños urbanos realizados a partir de arquitectos reconocidos a nivel internacional, b) las Villas Panamericanas denominado Proyecto Alameda, obra realizada para albergar a los deportistas durante los Juegos Panamericanos en el año 2011, c) Museo Gugenheim Guadalajara y d) Puerta Guadalajara, complejo que integra 16 edificios habitacionales, dos torres corporativas, dos museos, un centro comercial, un palacio de congresos, un hotel y áreas verdes (Rivera Borrayo y otros, 2009).

Por otro lado, Monterrey, Nuevo León se convirtió en un laboratorio para replicar las estrategias utilizadas en torno a megaproyectos urbanos en la Ciudad de México. Siendo uno de los estados más urbanizados con una reconocida área metropolitana integrada, ubicado en el norte del país (Aparicio Moreno y otros, 2011), presentó las condiciones necesarias para apostar a hacia las nuevas formas de hacer ciudad y la gestión urbana. A partir de los años noventa, surgen una serie de megaproyectos urbanos ubicados en diversas zonas de la ciudad, entre los cuáles surgen; a) Parque Fundidora, b) la renovación del centro histórico con el Proyecto Santa Lucia; c) el exclusivo conjunto urbanístico Valle Oriente y d) Ciudad Solidaridad (Garza y otros, 2003).

En resumen, los megaproyectos urbanos en México han configurado nuevos escenarios para las ciudades, a partir de generar nodos para la participación económica a nivel global mediante el fomento a la inversión, la diversificación de actividades y el desarrollo del turismo urbano. Los ejemplos emergen a lo largo del país.

\section{Hermosillo, Sonora}

Hermosillo, Sonora como un punto de partida para el estudio en torno a los megaproyectos urbanos. Capital del estado, colindante con el Mar de Cortés y con una franja 
fronteriza de 287 km con Estados Unidos; ubicación estratégica que le han brindado oportunidades para diversificar su economía en torno a la industria, el comercio, el turismo, la agricultura, la ganadería y la pesca.

Actualmente, Hermosillo se ha ubicado en el quinto lugar en el ranking de Ciudades con una población 500 mil a un millón de habitantes, de acuerdo al Instituto Mexicano para la Competitividad (IMCO, 2015). Para el cuál, se consideran algunos de los subíndices como: mercado laboral, estabilidad económica, gobiernos eficientes y eficaces, sociedad incluyente, preparada y sana, entre otros. Dichos cambios también se presentan como oportunidades para generar nuevos espacios urbanos en la ciudad acordes a las dinámicas a nivel nacional e internacional.

Hermosillo data sus orígenes en el siglo XVII a partir de los primeros asentamientos de los grupos indígenas de la región; Pimas, Seris y Tepocas. Para mediados del mismo siglo, se establecen los primeros presidios habitados por autoridades y soldados, así como por indígenas que eran reeducados a partir de las enseñanzas de la iglesia católica de la Colonia Española, Dicho presidio fue denominado como San Pedro de la Conquista del Pitic, Para el siglo XVIII, cambio su nombre a Villa del Pitic y es nombrada como cabecera de la región. A mitad del mismo siglo cambia su nombre a Hermosillo, siendo a su vez reconocida como capital del Estado de Sonora.

Su traza urbana a larga de su historia fue organizada en torno al cauce del río Sonora y a las inmediaciones del Cerro de la Campana. Durante el siglo XX, el desarrollo urbano de Hermosillo surge en torno a los proyectos de ciudad generados a partir del Porfiriato; periodo que aporto un significativo modelo de ciudad a partir de innovaciones tecnológicas en torno a la infraestructura, proyecto que sin duda apostaba a la modernidad urbana con fines económicos.
Para los años cuarenta, se presenta como una nueva oleada para el inconcluso proyecto urbano del Porfiriato. Hecho que vino a configurar nuevos espacios urbanos, así como parte de las avenidas y calles más importantes de la ciudad, así como la construcción de algunos de los edificios más emblemáticos, por ende, la construcción de la presa Abelardo L. Rodríguez. En los años sesenta, continua la fiebre constructiva a partir de dotar a la ciudad de vialidades y servicios públicos (Méndez, 2000).

\section{Megaproyectos urbanos en Hermosillo, Sonora}

1. Proyecto Especial Hermosillo. Desarrollo Urbano del Cauce del Río Sonora.

Hermosillo para finales de los años ochenta, comienza a presentar ciertas problemáticas urbanas derivadas de su morfología. Una de las características más representativas de Hermosillo, es el cauce del Río Sonora. Ubicado en el centro de la ciudad, se había convertido en una eminente amenaza para la población dado su posible desbordamiento, el cual podría haber causado la inundación de varias colonias aledañas. Así mismo, se había generado un mayor crecimiento hacia el norte, dejando el sur como una zona popular con poco abastecimiento en servicios públicos (Méndez, 2000).

La ciudad asumía retos económicos importantes tales como el establecimiento de la planta de ensamblaje de la Ford Company, la necesidad de fortalecer sus dinámicas regionales a partir generar una imagen urbana moderna, así como recuperar una zona central de la ciudad con el fin de consolidar un corredor económico, principalmente comercial y de servicios. Bajo este contexto de presura, para los últimos años del periodo gubernamental de Rodolfo Félix Valdez (1985-1991), se presenta el Proyecto Especial Hermosillo. Desarrollo Urbano del Cauce del Río Sonora. 
De acuerdo con el Ingeniero Cesar Silva Gómez, ex Secretario de Infraestructura y Desarrollo Urbano (19851991), "el proyecto cristalizo una necesidad añeja en la ciudad, así mismo abrió nuevas oportunidades para el desarrollo urbano. Pese a la negativa de algunos ciudadanos y el miedo al despojo, la obra fue consumada dejando en claro su eficiencia a los años, pues fue cuando pudimos percatarnos de el desfogue de la presa durante el gobierno de Beltrones, por lo que nos teníamos que la presa sobrepasará su capacidad..." (Fragmento de entrevista, junio 2016).

El proyecto se desarrolló en dos fases. En su primera fase (1987-1991), fueron obras de infraestructura e ingeniería urbana las que permitieron realizar la canalización del cauce del Río Sonora, la construcción de las compuertas en la presa Abelardo L. Rodríguez y la creación de la trama urbana en las zonas generadas a partir de la obra. Se construyeron alrededor de quince vialidades, siete puentes para el paso vehicular. Véase en el siguiente Mapa 1.

\section{MAPA 1. UBICACIÓN DEL PROYECTO}

\section{ESPECIAL HERMOSILLO. DESARROLLO URBANO DEL CAUCE DEL RÍO SONORA.}

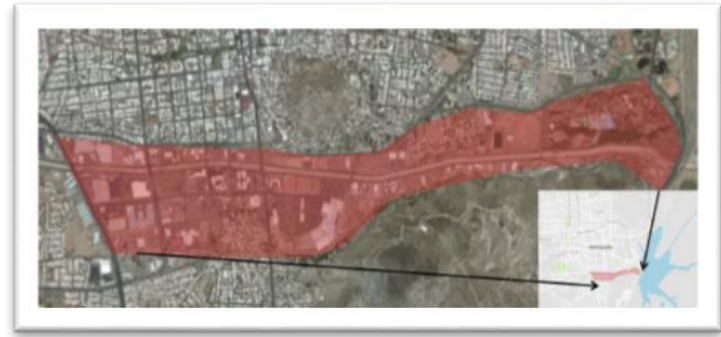

Fuente: Elaboración propia.

En una segunda fase (1991- 1997), se presenta el Plan Maestro Proyecto Río Sonora Hermosillo XX bajo la administración del entonces gobernador Manlio Fabio Beltrones Rivera. Esta propuesta apunta a capitalizar las obras de infraestructura generadas en la fase anterior con el fin de promover el desarrollo urbano en el denominado
Boulevard Río Sonora. Surgiendo un nuevo nodo urbano a partir de la creación de equipamientos recreativos, de gestión pública, bancarios, hospitalarios entre otros servicios y comercios (Méndez, 2000).

Este proyecto fue financiado bajo el fideicomiso Progreso Promotor Urbano de Sonora, el cual tenía la finalidad de modernizar la ciudad con el fin de convertirla en un centro urbano atractivo con equipamiento e infraestructura que permitiera atraer la inversión necesaria para generar progreso y modernización a la ciudad de Hermosillo y sus habitantes (Enríquez Acosta, 2002) Sin duda, ambos proyectos transformaron no solo la escena urbana, sino que incorporaron nuevas formas de gestión urbana a partir de la planificación estratégica y la aplicación incipiente de marketing urbano.

Entre las obras públicas que destacan durante este período, se encuentran la construcción del Centro de Gobierno del Estado de Sonora, integrado por dos edificios paralelos; Edificio Sonora y Edificio México, los cuáles comenzaron a albergar parte de las oficinas administrativas del gobierno estatal y federal. Así mismo, surge el Parque Recreativo La Sauceda. Dado a conocer como un proyecto innovador particularmente para el noroeste del país; el cuál incluía; áreas verdes, albercas con toboganes, canal (alimentado por agua de la presa) empleado para pasear en pequeñas lanchas, teatro al aire libre y el Museo La Burbuja. Véase Mapa 2.

\section{MAPA 2. UBICACIÓN DEL PLAN MAESTRO} PROYECTO RÍO SONORA HERMOSILLO SIGLO XX.

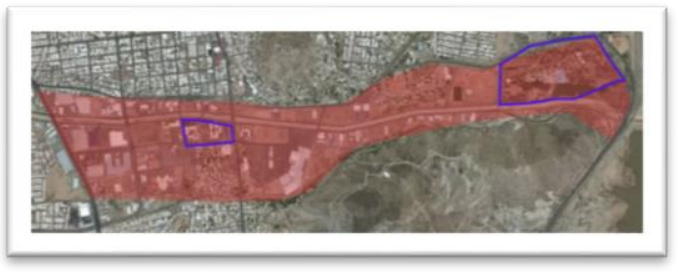

Fuente: Elaboración propia 
El Plan Maestro presentó algunos inciertos; a) la crisis de 1994 vino a paralizar parte de las obras urbanas generadas en torno al proyecto, provocando un incipiente inicio, b) la segregación socio-espacial continuó siendo un problema para la ciudad, sobre todo para la zona sur, c) el acaparamiento empresarial terminó por estipular la conformación del espacio urbano a partir de la inversión privada, y d) se popularizó el desarrollo de megaproyectos en otros sectores de la ciudad, vinculado a las elites económicas y políticas de la región: Entre estos últimos destacan; Metrocentro como un proyecto integrado por comercios y de servicios principalmente extranjeros, principalmente franquicias (Blockbuster, McDonald's, Domino's Pizza, entre otras). Así mismo, el residencial Los Lagos integrado por un club de golf, lagunas artificiales y mansiones de lujo (Méndez, 2000).

\section{Plan Sonora Proyecta}

Para el período administrativo gubernamental del Ing. Eduardo Bours Castelo (2004-2009), una de las principales apuestas para el desarrollo fue centrarse en grandes inversiones en materia de imagen $\mathrm{e}$ infraestructura urbana, con miras a generar estrategias de crecimiento económico en diversas regiones del Estado. Para poder orientar la política de gasto-inversión surge el Plan Sonora Proyecta con el objetivo de financiar más de 100 obras, y una inversión de casi 7 mil millones de pesos distribuidos en todo el estado de acuerdo a sus necesidades y prioridades especificas (Gobierno del Estado de Sonora, 2008). Véase Imagen 3.

A su vez, surge IMPULSOR Operadora de Proyectos Estratégicos como un fideicomiso para impulsar la infraestructura urbana de calidad mundial en zonas clave de Sonora. Los principales puntos de inversión fueron; Carretera Costera (Puerto Peñasco), Hermosillo, Guaymas, Pasajes (Rutas Turísticas) (Gobierno del Estado de Sonora, 2007b). Para la capital del estado, promovió una serie de complejos modernos y prácticos, que se integraban a la planeación urbana de la ciudad en condiciones de ordenamiento para los próximos veinticinco años (IMPULSOR, 2009).

Entre las intervenciones urbanas que se presentaron para Hermosillo, se encuentran las siguientes;

a. Hermosillo Puerta Norte

El proyecto partió en una primera fase; se presenta el plan parcial de desarrollo para la zona norte de la ciudad, mediante la prolongación de 6.5 kilómetros del Boulevard José María Morelos. Considerado como un importante corredor comercial y de servicios, la propuesta apuntaba a consolidar el sector urbano a partir de la inversión privada. (Gobierno del Estado de Sonora, 2007a). En una segunda fase, se agudiza más la perspectiva económica al señalar; a) la importancia de la promoción de la reserva territorial (2 mil 345 hectáreas) generada a partir de la prolongación del Boulevard Morelos, b) enfatiza el crecimiento ordenado así como la atracción de inversiones para la diversos sectores económicos sin señalar una articulación lógica urbana, y c) enfatiza en la conexión del Boulevard Morelos y Carretera Internacional como una obra de infraestructura que permitirá resolver los problemas de tráfico en la vieja salida norte de la ciudad (Gobierno del Estado de Sonora, 2008). Véase Mapa 3.

\section{MAPA 3. HERMOSILLO PUERTA NORTE.}

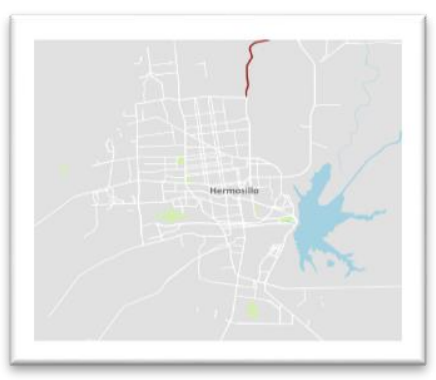

Fuente: Elaboración propia. 
b. Hermosillo Vado del Río

El siguiente proyecto busca capitalizar una vez más el Vado del Río o bien conocido, como Plan Maestro Río Sonora Siglo XXI. Pesé a los años, el Boulevard Río Sonora había podido ocupar sólo el $48 \%$ de los terrenos disponibles. La consolidación de la zona como uno de los corredores comerciales y de servicios de la ciudad se había quedado suspendida, por tal motivo era necesaria rehabilitar las zonas existentes a partir inversiones por medio de la promoción de los espacios urbanos restantes entre el sector empresarial (Gobierno del Estado de Sonora, 2008).

Las acciones para cumplir los objetivos se plantearon a partir tres puntos; redefinir el plan maestro con el fin de establecer polígonos a partir de las directrices del mercado, aumentar la plusvalía a partir de mejorar la imagen urbana y la circulación en vialidades y por último, impulsar el desarrollo de proyectos detonadores (Impulsor 2007).

Entre los proyectos a desarrollarse en torno a los polígonos comerciales y de servicios se encontraban: Hotel Camino Real y Hospital Ángeles, Gallerías Mall, Soriana del Río, Hotel Lucerna, Hotel City Express, Famsa, Banamex y Pabellón Reforma. La inversión total a concretarse entre el 2007 y 2008, sumaban alrededor de 2, 543.6 millones de pesos. Así como el valor del suelo se ubicaba entre 80 a 200 dólares por metro cuadrado en torno al Boulevard Río Sonora (Manjarrez, 2006). Véase en la siguiente Mapa 4.

\section{MAPA 4. HERMOSILLO VADO DEL RÍO. GALLERÍAS MALL Y MUSAS.}

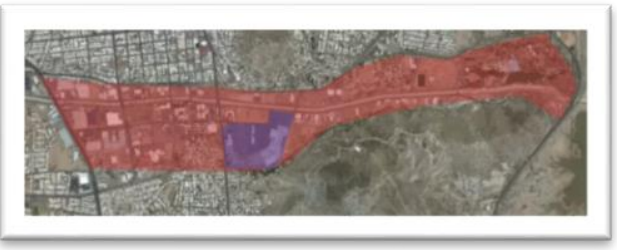

Fuente: Elaboración propia
El proyecto detonador de Hermosillo Vado del Río giro en torno al Centro Cultural MUSAS, el cuál consistía en un complejo cultural con el propósito de crear espacios para la formación, difusión y promoción del arte y la cultura en Sonora. Este estaría integrado por: 1) Teatro de usos múltiples, 2) Museo, 3) Centro del Visitante y 4) Plaza de la cultura (IMPULSOR 2007). Las inversiones se estimaban alrededor de 25 millones 114 mil de pesos (Gobierno del Estado de Sonora, 2008).

Entre otros de los proyectos públicos en torno al Boulevard Paseo Río Sonora surge Parque Conmemorativo del Centenario de la Revolución, con una inversión de 3.1 millones de pesos. El proyecto se pensó a partir de un andador peatonal, áreas de mesas y bancas, juegos infantiles, estacionamiento y áreas verdes (Gobierno del Estado de Sonora, 2009a).

\section{Proyecto Puerta Oeste Hermosillo}

Para la siguiente administración gubernamental a cargo del Lic. Guillermo Padrés Elías (2009-2015), uno de los objetivos para Sonora sería la transformación económica a partir del desarrollo urbano, principalmente de su capital, Hermosillo. Por tal motivo, para el 2010 se da a conocer el Proyecto Puerta Oeste Hermosillo, el cuál buscaba generar un modelo de desarrollo con visión a futuro en el que convergieran obras comerciales, de vivienda y deportivas. El proyecto nuevamente se apoyó en IMPULSOR como principal promotor urbano.

El proyecto se planteaba como una estrategia urbana que pudiera ser replicada en las principales ciudades del estado; Ciudad Obregón, Navojoa y Nogales. El complejo se encontraba integrado por espacios urbanos destinados para vivienda económica, media y residencial, así como áreas públicas para el deporte y el esparcimiento familiar. También incluía escuelas, jardines, hospitales, bancas comerciales, entre otros.

El proyecto fue diseñado tomando como punto de partida el "Estadio Sonora", diseñado con la más alta tecnología 
con el objetivo de ser anfitrión de partidos de las grandes ligas de beisbol, y por ende ser la casa del equipo local "Naranjeros de Hermosillo" (Gobierno del Estado de Sonora 2011b). Observar en el Imagen 2.

\section{IMAGEN 2. PROYECCIONES DEL ESTADIO} SONORA.

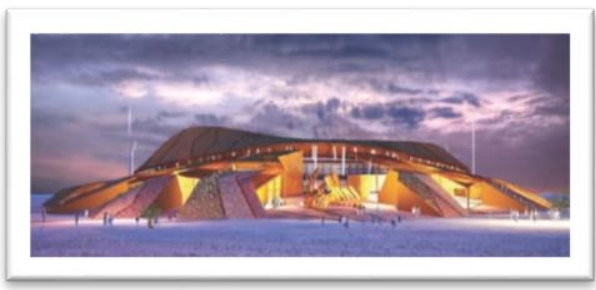

Fuente: Gobierno del Estado de Sonora (2011c).

Durante el mismo período se realizó el cambio al Programa de Desarrollo Urbano para el Centro de Población de Hermosillo 2014, el cual presentaba algunos ligeros cambios con respecto al presentado en el 2007. No obstante, fue reelaborado con el fin de dar respaldo al Proyecto Puerta Oeste a partir de los cambios realizados en la Ley de Ordenamiento Territorial y Desarrollo Urbano del Estado de Sonora. Véase Mapa 5.

Nuevamente el Proyecto Puerta Oeste vino a presentar los mismos vicios; reportes financieros poco explícitos o nulos, concesiones realizadas a familiares o amigos del Gobernador y, el crecimiento de la deuda pública. Estos hechos para algunos de los ciudadanos no pudieron romper con la tradición beisbolera y dejar de lado el orgullo sonorense al incorporar un nuevo recinto para la actividad deportiva.

\section{MAPA 5. UBICACIÓN DEL PROYECTO} PUERTA OESTE.

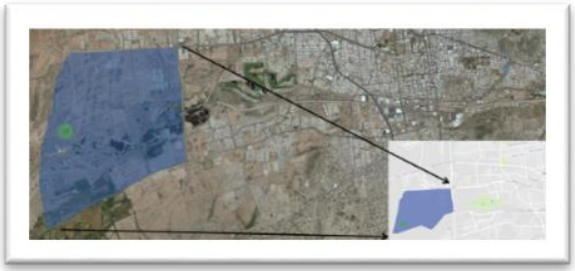

Fuente: Elaboración propia.

\section{Consecuencias urbanas}

Dentro de las consecuencias urbanas generadas a partir de la introducción de los megaproyectos urbanos en Hermosillo, Sonora ha detonado la inversión privada particularmente vinculado a zonas residenciales. Se calcula que existen alrededor de 28 zonas residenciales de alta y media plusvalía en las áreas generadas a partir de los proyectos. Véase en el siguiente Cuadro 1.

Las zonas de vivienda particularizan por tener grandes extensiones de terreno, así como edificaciones que no resultan sustentables para el medio ambiente sonorense, entre ellas, la construcción de albercas para áreas comunes. Así mismo la sobre construcción de fraccionamientos residenciales cerrados ha dado como resultado la exacerbación de zonas de pobreza aledañas, así como la disputa por la falta de servicios básicos e inseguridad.

La promoción de la vivienda bajo las particularidades de fraccionamientos cerrados ha venido a estimular un gran mercado a su vez de plazas comerciales que son dotadas de comercios y servicios tanto locales, nacionales como internacionales. La distribución del espacio se plantea como una disputa por obtener el mercado que sale de las principales residenciales cerradas. 
CUADRO 1. MEGAPROYECTOS URBANOS EN HERMOSILLO, SONORA

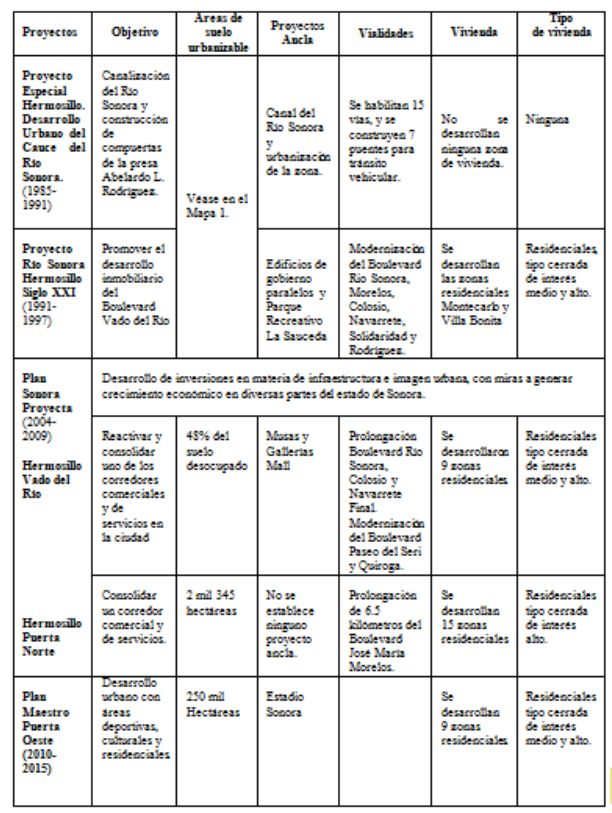

Fuente: Elaboración propia.

Sin duda, la privatización a partir de la generación de megaproyectos urbanos ha generado grandes rezagos urbanos en otras zonas de la ciudad, se ha olvidado el centro histórico como uno de los principales motores económicos. En donde el comercio se convierte en una experiencia de poca valía y con nulas condiciones para sus transportarse por sus vialidades y escasos estacionamientos.

Por otro lado, las viviendas en las zonas centrales en la ciudad se encuentran sujetas a un proceso de abandono debido a la precariedad en servicios públicos y atención en temas urbanos, como principalmente la inseguridad. Las condiciones de las viejas casonas se convierten en un problema para el patrimonio urbano, dado que consecuentemente son derrumbadas, dadas el abandono de sus dueños.

La falta de mecanismos que permitan dar un seguimiento a los megaproyectos urbanos no es sólo una de las problemáticas, sino su falta de capacidad para poder integrar otras zonas de la ciudad que carecen o que prácticamente necesitan más que otras zonas de la ciudad, como la falta de pavimentación de calidad y particularmente la seguridad.

La diversidad de problemáticas urbanas se acentúa entre la presura por la construcción de proyectos que detonen la económica y relumbren en la escena estatal, así como la conveniencia de hacer cada vez más difícil los caminos para transparentar los gastos públicos generados en cada uno de los proyectos.

Es difícil llegar a terminar de concebir cada uno de los escenarios bajo los cuáles la ciudad se construye, no obstante, las consecuencias son presentes y dejan cada vez más a los ciudadanos sin espacios públicos. El peso de las grandes empresas ha dejado de lado, la capacidad de las estancias municipales y estatales de establecer espacios que brinden la seguridad y el confort necesario para desarrollar actividades al aire libre.

En suma, los megaproyectos urbanos se convierten en una posible forma de hacer ciudad siempre y cuando sus condiciones sean cumplidas. La necesidad de espacios urbanos de calidad se convierte en uno de los temas en discusión más acentuado en nuestra actualidad, por lo cual no hay que olvidar la capacidad de los gobiernos para dar respuestas y la voluntad de sus ciudadanos para solicitarlas.

\section{Conclusiones}

Los megaproyectos urbanos se han establecido como una forma de hacer ciudad, si bien desde su definición podemos argumentar que integran una serie de intervenciones urbanas que surgen de la iniciativa pública y privada (Cuenya 2009), las cuáles encierran una serie de usos y funciones jerárquicos del espacio, generando nuevas centralidades derivadas de su localización estratégica aunadas a la demanda del poder adquisitivo más alto. Sus tipologías se expanden a operaciones no 
sólo a zonas de renovación y rehabilitación urbana sino considera el montaje de infraestructura (edificios) con el fin de albergar grandes eventos de índole cultural y deportivo (Cuenya y otros, 2012).

La búsqueda de multiplicidad de funciones en los megaproyectos urbanos conlleva a espacios urbanos cada vez más complejos, las cuáles adquieren sentido a partir de una obra arquitectónica central. Estos complejos urbanos que intentan integrar zonas residenciales, comerciales, deportivas y de ocio vienen a conformar nichos económicos con el fin de seguir fomentando la inversión inmobiliaria en la ciudad. Su integración a la trama urbana viene gestionándose a partir de aplicar estrategias de planificación y marketing urbano, a partir de esferas públicas como privadas.

En ese sentido, la planificación estratégica funciona como una forma de gestionar la ciudad desde la mirada empresarial, en donde se busca mantener una postura abierta a los cambios y por ende a la toma de decisiones al momento de hacer ciudad (Fernández Güell, 2004) De acuerdo a Borja y Castells (1998), la planificación estratégica debe girar en torno a un proyecto de ciudad para cuál se establezcan marcos de acción claros y precisos. Por ende, es necesario definir un proyecto de ciudad con el fin de generar la adhesión colectiva de la población, y no sólo de los sectores empresariales.

Así mismo la planificación estratégica se presenta en la práctica a partir de planteamientos que inducen a señalar tan solo las fortalezas y las debilidades de un marco territorial para su promoción. Por otro lado, los marcos normativos de regularización del suelo terminan por operar a partir de las fuerzas del mercado, hecho que conduce a consolidar grupos de poder, tanto dentro como fuera de aparato gubernamental (Peña Medina, 2016). Tras esta redefinición operativa de las ciudades se encuentra una serie de condiciones a nivel global en torno a las cuáles se buscan posicionarse por medio como producto más dentro del ejercicio del capitalismo, tal motivo es necesario consolidar una o varias imágenes urbanas poder medio de las cuáles hacer efectiva la planificación estratégica en un entorno de mercados competitivos, que buscan espacios para el desarrollo de capitales a partir de la inversión inmobiliaria (Precedo Ledo, 1996).

Mientras el marketing urbano surge como un ejercicio que permite dar a conocer las ventajas y las desventajas de conformar un proyecto de ciudad en torno a un concepto definido de ciudad. De acuerdo a Muxí (2004), estamos invadidos por grandes proyectos de marketing que intentan dar soporte a ciertos momentos de la historia en la sociedad moderna. De tal forma, la ciudad no escapa a la búsqueda de mecanismos de persuasión en torno a que sus capacidades y sus formas son más prosperas que otras.

En el caso de Hermosillo, los megaproyectos urbanos se han integrado a la planificación estratégica a partir de la generación de modelos urbanos que han permitido gestionar el uso del suelo de acuerdo a las demandas del mercado, dando lugar al a hegemonía del capital privado, principalmente en la conformación de zonas residenciales y comerciales que emergen en torno a los grandes proyectos urbanos (Abramo, 2012).

No obstante, los megaproyectos urbanos no han logrado definir un objetivo en común o bien, un proyecto de ciudad que permita dar pauta a las intervenciones urbanas para un proyecto a largo plazo. Por el contrario, su condición conceptual ha sido dispersa, sin un sustento discursivo que permita integrar en una idea del rumbo de la ciudad. Por consecuencia no ha sido posible diseñar una marca de ciudad que pueda representar la oferta de la misma, y por ende una estrategia de marketing urbano concreta.

Los mecanismos empleados en torno a marketing urbano han sido basados en el convencimiento explícito de la 
benevolencia de los megaproyectos urbanos. Hecho que ha dejado de lado cualquier forma de participación ciudadana que permita dar a conocer las necesidades, los intereses y los deseos de quienes habitan la ciudad. Esto sin duda, se convierte en una de las principales consecuencias de la planificación urbana apoyada en megaproyectos.

Por otro lado, la proliferación de plazas comerciales, residenciales en modelo de cerradas, así como la privatización del espacio público ha influido en las formas de concebir e integrarse al imaginario urbano de la ciudad. Hecho que sin duda viene a volcar las pocas posibilidades de conformar agrupaciones ciudadanas preocupadas por el desarrollo equitativo de la ciudad.

En suma, resulta necesario reconsiderar la gestión urbana desde principios que permitan generar no sólo un polo de atracción para las inversiones, sino desde una lógica que torne a las necesidades de los ciudadanos como principal punto de partida. Sin embargo, mientras los mecanismos de acumulación y generación de capitales sean los mismos, nos seguiremos enfrentando a las mismas problemáticas en cada una de las ciudades que analicemos.

\section{Bibliografía}

ABRAMO, P. (2012). "La ciudad com-fusa: mercado y producción de la estrucura urbana en las grandes metrópolis latinoamericanas." Eure, Vol .38 Núm.114, Mayo, pp.35-69. (14 de Septiembre del 2016)

APARICIO MORENO, C. E., ORTEGA RUBÍ M. E. y E. S. HERNÁNDEZ. (2011). "La segregación socio-espacial en Monterrey a lo largo de su proceso de metropolización.” Revista Región y Sociedad. Vol. 23, Núm. 52. Septiembre-diciembre, pp. 173-207. (14 de Septiembre del 2016)

BORJA, J. y CASTELLS, M. (1998), Local y global. La gestión de las ciudades en la era de ia información. México: Taurus.

CUENYA, B., NOVÁIS, P. y VAINER, C. (2012), Grandes proyectos urbanos. Miradas sobre la experiencia argentina y brasileña. Buenos Aires: Cafe de las ciudades. (14 de Septiembre del 2016)

CUENYA, B. (2009). "Grandes proyectos urbanos latinoamericanos. Aportes para su conceptualización y gestión desde la perspectiva del gobierno local." Cuaderno Urbano. Espacio, cultura, sociedad. Vol. 8, Núm. 8, Octubre, pp. 229-252. (14 de Septiembre del 2016)
DÍAZ ORUETA, F. (2009), "El impacto de los megaproyectos en las ciudades españolas. Hacia una aganda de investigación." Estudios Demográficos y Urbanos, Vol. 24, Núm.1,enero-abril, pp.193-218. (14 de Septiembre del 2016)

DÍAZ ORUETA, F. (2015), "Megaproyectos urbanos y modelo de ciudad. El ejemplo de Madrid Río." Cuaderno Urbano. Espacio, cultura, sociedad, Vol.19, Núm.19, Diciembre, pp.179-200. (14 de Septiembre del 2016)

ENRIQUEZ ACOSTA, J. A. (2002), El Progreso Hermosillense. Expresiones de la modernización en los años noventa del Siglo XX. Colección Textos Académicos. Universidad de Sonora. Hermosillo, Sonora, México.

FAINSTEIN, S. S. (2009). "Megaprojects New York, London y Amsterdam." International Journal of Urban and Regional Research. Vol. 32, Núm. 4, pp. 768-785. (14 de Septiembre del 2016)

FERNANDEZ GUELL., J. M. (2004), Planificación estratégica de las ciudades. Barcelona: Gustavo Gili.

GARZA, G., PIERRE F. y GARY S. (2003). Políticas urbanas en grandes metrópolis: Detroit, Monterrey y Toronto. Programa Interinstitucional de Estudios sobre la Región de América del Norte. El Colegio de México. Ciudad de México, México.

GOBIERNO DEL ESTADO DE SONORA (2007a), Informe de Gobierno. Hermosillo, Sonora: Gobierno del Estado de Sonora. (14 de Septiembre del 2016)

GOBIERNO DEL ESTADO DE SONORA (2007b), Presentación de Impulsor Sonora. Hermosillo, Sonora, México. (14 de Septiembre del 2016)

GOBIERNO DEL ESTADO DE SONORA (2008), Informe de Gobierno. Hermosillo, Sonora: Gobierno del Estado de Sonora. (14 de Septiembre del 2016)

GOBIERNO DEL ESTADO DE SONORA (2009), Informe de Gobierno. Hermosillo, Sonora: Gobierno del Estado de Sonora. (14 de Septiembre del 2016)

GOBIERNO DEL ESTADO DE SONORA (2011), Comunicado de prensa. Hermosillo, Sonora, México: Gobierno del Estado de Sonora. (14 de Septiembre del 2016)

IMPULSOR (2007). Presentación MUSAS. Gobierno del Estado de Sonora. (14 de Septiembre del 2016)

IMPULSOR (2009). Presentación MUSAS. Musas. Música, Arte y Exposiciones en Sonora. Hermosillo, Sonora , México: Gobierno del Estado de Sonora. (14 de Septiembre del 2016)

IMCO (2015). Indice de Ciudades Competitivas y Sustentables. http://imco.org.mx/competitividad/indice-de-ciudades-competitivas-ysustentables-2015/ (4 de diciembre del 2016)

LUNGO, M. (2005a), Globalización, grandes proyectos y privatización de la gestión urbana. (U. d. Bío, Ed.) Urbano,Vol. 8, Núm. 11, pp. 49-58. (14 de Septiembre del 2016)

LUNGO, M. (2005b), Grandes proyectos urbanos. Una visión general. Urbana, Vol.10, Núm.37, pp. 15-46. (14 de Septiembre del 2016) 
MANJARREZ, A. (2006). Revive zona del vado del rpio. El Imparcial Sonora, Sección A Mercados. (14 de Septiembre del 2016)

MORENO CARRANCO, M. (2008). "La producción espacial de lo global: lo público y lo privado en Santa Fe, Ciudad de México." Revista Alteridades. Vol.18, Núm. 36, julio-diciembre pp. 75-86. (14 de Septiembre del 2016)

MÉNDEZ, E. (2000). Hermosillo en el Siglo XX. Urbanismos incompletos y arquitecturas emblemáticas. Hermosillo, Sonora, El Colegio de Sonora.

MUXÍ, Z. (2004). La arquitectura de la ciudad global. Barcelona, Gustavo Gili.

PEÑA MEDINA, S. (2016), Teoría, procesos y práctica de la planeación urbana y regional. Tijuana, Baja California Norte: El Colegio de la Frontera Norte.

PRADILLA COBOS, Emilio. ( 2004), "Ciudad de México: los caminos de la privatización de lo urbano." Revista Ciudades 64. Vol.16, OctubreDiciembre, pp. 57-62. Puebla, (14 de Septiembre del 2016)

PRECEDO LEDO, A. (1996), Ciudad y Desarrollo Urbano. Madrid, España: Síntesis.

RIVERA BORVAYO, E. y OROZCO ALVARADO J. (2009), "Grandes proyectos de intervención en la ciudad-metrópoli: Guadalajara, México y los retos en la planeación urbana."Acta de Quinto Congreso Internacional Ciudad Virtual y Territorio en Junio 2, 3 y 4 del 2009. Universidad de Politécnica Catalunya. Disponible en http://upcommons.upc.edu/handle/2099/11960;jsessionid=F9A0B45A362 0BE02A81D703708623E3C (10 de Septiembre del 2016)

TALLEDOS SANCHEZ. E. y IBARRA GARCÍA M. V. (eds.) (2016), Megaproyectos en México. Una mirada crítica. México, Itaca.

VALENZUELA, A. (2013). "Dispositivos de la globalización: la construcción de grandes proyectos urbanos en Ciudad de México." Revista EURE. Vol. 39, Núm. 116, julio-diciembre pp. 101-118. (14 de Septiembre del 2016)

VALENZUELA, A. (2007). "Santa Fé (México): Megaproyectos para una ciudad dividida." Cuadernos Geográficos. Departamento de Análisis Geográfico Regional y Geografía Física. Departamento de Geografía Humana. Revistas de la Universidad de Granada. Núm. 40. Semestral, pp.53-66. (14 de Septiembre del 2016 
